\title{
Hopf Bifurcation Analysis and Anticontrol of Hopf Circles of the Rössler-Like System
}

\author{
Ranchao Wu and Xiang Li \\ School of Mathematics, Anhui University, Hefei 230039, China \\ Correspondence should be addressed to Ranchao Wu, rcwu@ahu.edu.cn
}

Received 15 March 2012; Accepted 20 June 2012

Academic Editor: Allan Peterson

Copyright (C) 2012 R. Wu and X. Li. This is an open access article distributed under the Creative Commons Attribution License, which permits unrestricted use, distribution, and reproduction in any medium, provided the original work is properly cited.

A new Rössler-like system is constructed by the linear feedback control scheme in this paper. As well, it exhibits complex dynamical behaviors, such as bifurcation, chaos, and strange attractor. By virtue of the normal form theory, its Hopf bifurcation and stability are investigated in detail. Consequently, the stable periodic orbits are bifurcated. Furthermore, the anticontrol of Hopf circles is achieved between the new Rössler-like system and the original Rössler one via a modified projective synchronization scheme. As a result, a stable Hopf circle is created in the controlled Rössler system. The corresponding numerical simulations are presented, which agree with the theoretical analysis.

\section{Introduction}

In the last three decades, chaos has been studied extensively and attracted increasing interests from mathematicians, physicists, engineers, and so on. Since chaotic systems not only admit abundant complex and interesting dynamical behaviors, such as bifurcations, chaos, and strange attractors, but also have many potential practical applications, great efforts have been devoted to investigating chaotic systems, for example, Lorenz system [1] and Rössler system $[2,3]$, and there has been an increasing effort to construct different types of chaotic systems. During the last few years, some new Lorenz-like chaotic systems [4], including Chen system [5], Lü system [6], Liu system [7], and T system [8], were proposed and studied.

Research on bifurcation, such as Hopf bifurcation, homoclinic bifurcation, and period doubling bifurcation, is one of the most hot topics in the field of nonlinear science [9]. It has been found that bifurcation will frequently lead to chaos in nonlinear systems. So it is necessary to explore the bifurcation of dynamical systems so as to understand the complex dynamical behaviors. Recently, Hopf bifurcation of some famous chaotic systems has been investigated and it has been becoming one of the most active topics in the field of chaotic systems. 
In this paper, a new Rössler-like system is constructed by adding a linear feedback control term to the original Rössler system [2]. It is found that the new system admits complex dynamical behaviors with varying parameters, such as bifurcation, chaos, and strange attractors. Now its basic dynamical behaviors are analyzed, especially its Hopf bifurcation is investigated in detail via theoretical as well as numerical analysis. The bifurcated periodic solutions will be created under some parameter conditions.

As is well known, Hopf bifurcation gives rise to limit circles, which are typical oscillatory behaviors of many nonlinear systems in physical, social, economic, biological, and chemical fields. These oscillatory behaviors [10-12] can be beneficial in practical applications, such as in mixing, monitoring, and fault diagnosis in electromechanical systems. Also, the properties of limit cycles are very useful in modern control engineering, such as autotuning of PID controller [13] and process identification [14]. Early efforts in Hopf bifurcation control focused on delaying the onset of this bifurcation [15] or stabilizing an existing bifurcation [16]. In this paper, we propose a control method based on modified projective synchronization (MPS) [17] to generate a Hopf limit circle in the original Rössler system. The proposed MPS involves less calculations. Furthermore, the shape and size of the created Hopf circle can be adjusted by choosing different scaling factors.

The paper is organized as follows. In Section 2, a new Rössler-like system is constructed by the linear feedback control. In Section 3, its dissipation and fractal dimension are analyzed. The local stability and existence of Hopf bifurcation, as well as the direction and stability of bifurcating periodic solutions, are discussed in detail by the normal form theory in Section 4. In Section 5, the anticontrol of Hopf circles is achieved between the new Rössler-like system and the original Rössler system via the modified projective synchronization scheme, and a stable Hopf limit circle is created in the original Rössler system successfully. Finally, some conclusions are drawn in Section 6.

\section{The New Modified Rössler System}

The Rössler system [2] is described by

$$
\begin{gathered}
\dot{x}=-y-z, \\
\dot{y}=x, \\
\dot{z}=a\left(y-y^{2}\right)-b z,
\end{gathered}
$$

which is chaotic when $a=b=0.5$, and its strange attractor is shown in Figure 1 .

Based on this Rössler system, by adding a linear term to the second equation and changing the third equation of system (2.1), a new Rössler-like system is obtained and given by

$$
\begin{gathered}
\dot{x}=-y-z, \\
\dot{y}=x+c z, \\
\dot{z}=a y-b z-y^{2},
\end{gathered}
$$

where $x, y, z$ are state variables, and $a, b, c$ are parameters. In order to ensure that system (2.2) is a dissipative system, assume that the parameter $b$ is positive in the following discussions. 


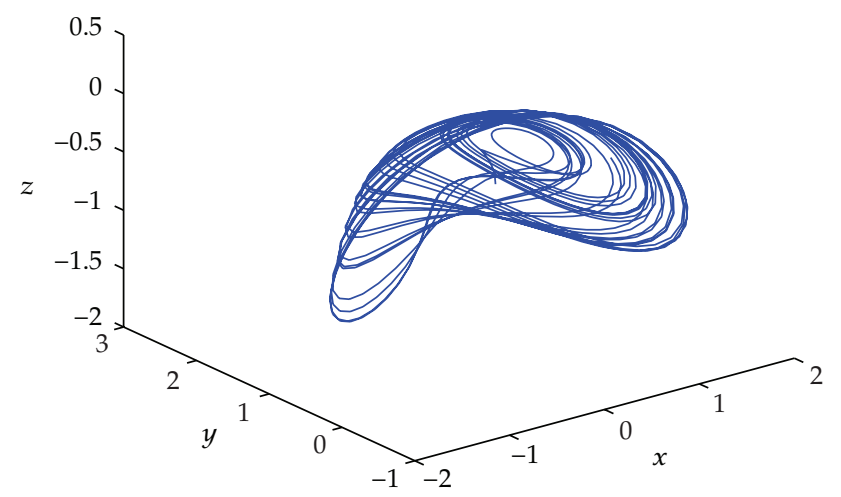

Figure 1: Chaotic attractor of system (2.1).

When $a=0.5, b=1, c=1.2$, system (2.2) is chaotic, which is shown in Figure 2. The bifurcation diagram of state variable $y$ versus parameter $c$ is shown in Figure 3. Figure 4 is the state trajectory of $y$.

\section{Analysis of Basic Dynamic Behaviors}

\subsection{Dissipation and Existence of Attractor}

The divergence of system (2.2) is defined by

$$
\nabla V=\frac{\partial \dot{x}}{\partial x}+\frac{\partial \dot{y}}{\partial y}+\frac{\partial \dot{z}}{\partial z}=-b=-1<0
$$

Since $\nabla V<0$, system (2.2) is a dissipative system and converges with an index rate of $e^{-t}$. Volume element $V_{0}$ shrinks to $V_{0} e^{-t}$ at the time $t$. When $t \rightarrow \infty$, volume element $V_{0}$ shrinks to 0 . Therefore, all trajectories of system (2.2) will be confined to a congregation, whose volume is 0 . Its gradual movement behaviors are fixed in an attractor.

\subsection{The Lyapunov Dimension}

As we know, the Lyapunov exponents measure the exponential rates of divergence or convergence of nearby trajectories in phase space. Using Matlab software, the three Lyapunov exponents of system (2.2) are, respectively, $\lambda_{1}=0.0701, \lambda_{2}=0, \lambda_{3}=-1.0796$ when $a=0.5, b=$ $1, c=1.2$. The Lyapunov dimension of chaotic attractor of this new Rössler-like system is fractional, which is described as

$$
D_{L}=j+\frac{1}{\left|\lambda_{j+1}\right|} \sum_{i=1}^{j} \lambda_{i}=2+\frac{1}{|-1.0796|}(0.0701+0)=2.0649
$$




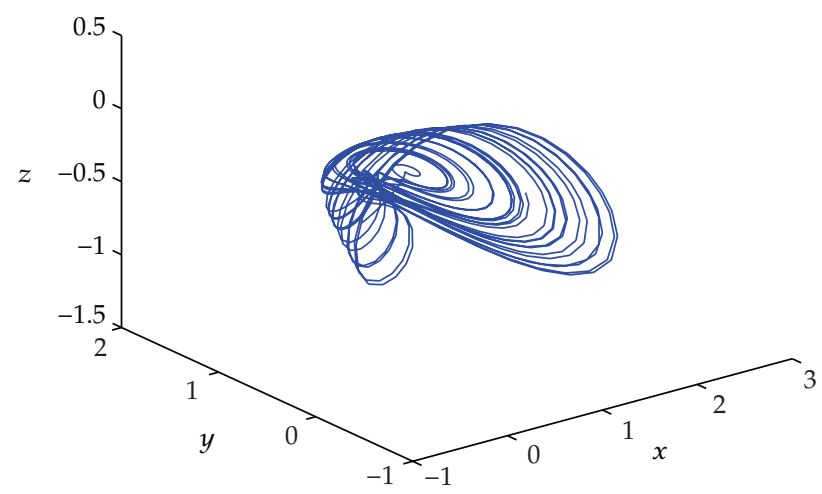

Figure 2: Chaotic attractor of system (2.2).

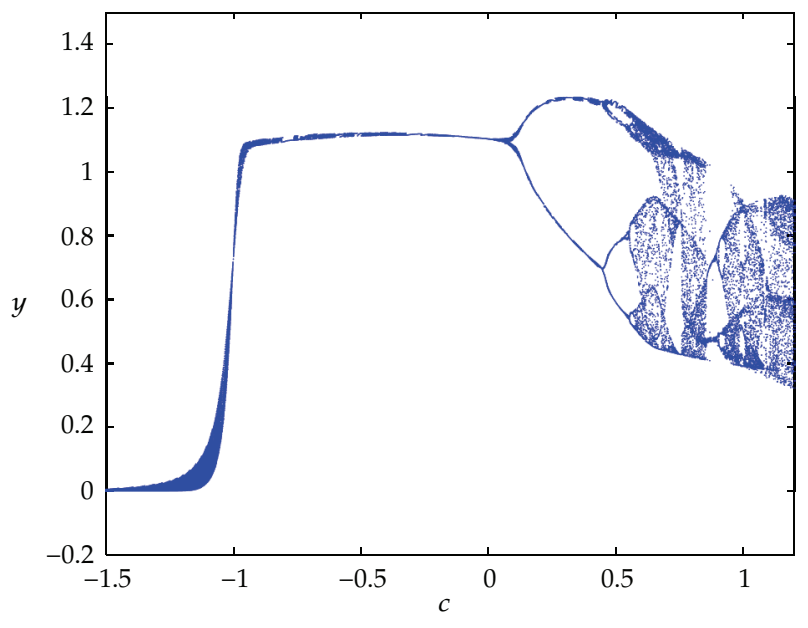

Figure 3: Bifurcation diagram of system (2.2).

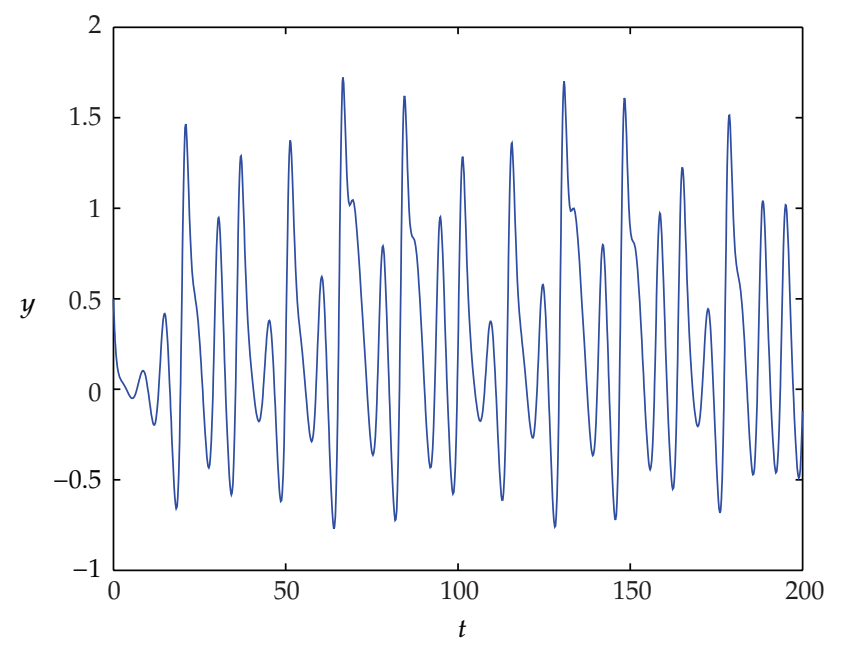

Figure 4: State trajectory of $y$. 


\subsection{Local Stability}

By simple computation, it is easy to obtain that system (2.2) has two equilibria $O(0,0,0)$ and $E\left(c y_{0}, y_{0},-y_{0}\right)$, where $y_{0}=a+b$. The Jacobian matrix for system (2.2) at the equilibrium $O(0,0,0)$ and the equilibrium $E\left(c y_{0}, y_{0},-y_{0}\right)$ are, respectively, given by

$$
J=\left(\begin{array}{ccc}
0 & -1 & -1 \\
1 & 0 & c \\
0 & a & -b
\end{array}\right), \quad \tilde{J}=\left(\begin{array}{ccc}
0 & -1 & -1 \\
1 & 0 & c \\
0 & -a-2 b & -b
\end{array}\right)
$$

and their corresponding characteristic equation are, respectively,

$$
\begin{gathered}
f(\lambda)=\lambda^{3}+b \lambda^{2}+(1-a c) \lambda+a+b=0, \\
\tilde{f}(\lambda)=\lambda^{3}+b \lambda^{2}+(1+2 b c+a c) \lambda-a-b=0 .
\end{gathered}
$$

According to Routh-Hurwitz's criterion, the real parts of the roots of (3.4) are all negative if and only if

$$
b>0, \quad a+a b c<0, \quad a+b>0,
$$

and the real parts of the roots of (3.5) are all negative if and only if

$$
b>0, \quad(b c+1)(a+2 b)>0, \quad a+b<0 .
$$

The above analysis is summarized as follows.

Theorem 3.1. For the two equilibria $O$ and $E$ of the new Rössler-like system (2.2),

(1) when $b>0, a+a b c<0, a+b>0$, the equilibrium $O$ is asymptotically stable.

(2) when $b>0,(b c+1)(a+2 b)>0, a+b<0$, the equilibrium $E$ is asymptotically stable.

\section{Hopf Bifurcation Analysis of the New System}

\subsection{Hopf Bifurcation Analysis about $O(0,0,0)$}

Let us assume that the solutions to the new Rössler-like system (2.2) undergo a Hopf bifurcation on some submanifold in parameter space corresponding to fixed $c=c_{0}$. Then the characteristic equation (3.4) has roots $\lambda_{0} \in R$ and $\lambda_{ \pm}= \pm i \omega_{0}$, where $\omega_{0} \in R^{+}$. Then, one can obtain that

$$
f(\lambda)=\left(\lambda-\lambda_{0}\right)\left(\lambda^{2}+\omega_{0}^{2}\right)=\lambda^{3}+b \lambda^{2}+(1-a c) \lambda+a+b .
$$


Clearly,

$$
\lambda_{0}=-b, \quad \omega_{0}^{2}=1-a c, \quad-\lambda_{0} \omega_{0}^{2}=a+b .
$$

From (4.2), it is easy to get that (3.4) has a pair of purely imaginary conjugate roots $\lambda_{ \pm}=$ $\pm i \sqrt{1+a / b} \equiv \pm i \omega_{0}$ and a real root $\lambda_{0}=-b$ if and only if

$$
c=c_{0}=\frac{-1}{b}, \quad 1+\frac{a}{b}>0
$$

According to (3.4), one has

$$
\lambda^{\prime}(c)=\frac{a \lambda}{3 \lambda^{2}+2 b \lambda-a c+1} .
$$

Therefore,

$$
\operatorname{Re}\left(\lambda^{\prime}\left(c_{0}\right)\right)=\frac{a b^{3} \omega_{0}^{2}}{2(a+b)^{2}+2 b^{4} \omega_{0}^{2}}
$$

By Hopf bifurcation theorem [18] and the above analysis, we have the following

Theorem 4.1 (Existence of Hopf bifurcation). When $a \neq 0, b>0, a+b>0$ and $c$ passes through the critical value $c_{0}=-1 / b$, system (2.2) undergoes a Hopf bifurcation at the equilibrium point $\mathrm{O}(0,0,0)$.

\subsection{Direction and Stability of Bifurcating Periodic Orbits}

In this section, we apply the normal form theory [18] to study the direction, stability, and period of bifurcating periodic solutions for system (2.2).

The eigenvectors $v_{1}, v_{0}$ associated with $\lambda_{+}=i \omega_{0}, \lambda_{0}=-b$ are, respectively,

$$
v_{1}=\left(\begin{array}{c}
-\frac{1}{a}-\frac{\sqrt{b^{2}+a b}}{a} i \\
\frac{b}{a}-\frac{\sqrt{b^{2}+a b}}{a b} i \\
1
\end{array}\right), \quad v_{0}=\left(\begin{array}{c}
\frac{1}{b} \\
0 \\
1
\end{array}\right)
$$




\section{Define}

$$
\begin{gathered}
P=\left(\operatorname{Re} v_{1}, \operatorname{Im} v_{1}, v_{0}\right)=\left(\begin{array}{ccc}
-\frac{1}{a} & -\frac{\sqrt{b^{2}+a b}}{a} & \frac{1}{b} \\
\frac{b}{a} & -\frac{\sqrt{b^{2}+a b}}{a b} & 0 \\
1 & 0 & 1
\end{array}\right), \\
(x, y, z)^{T}=\left(x_{1}, y_{1}, z_{1}\right)^{T} .
\end{gathered}
$$

Then system (2.2) can be written into

$$
\begin{gathered}
\dot{x}_{1}=-\omega_{0} y_{1}+F_{1}\left(x_{1}, y_{1}, z_{1}\right), \\
\dot{y}_{1}=\omega_{0} x_{1}+F_{2}\left(x_{1}, y_{1}, z_{1}\right), \\
\dot{z}_{1}=-b z_{1}+F_{3}\left(x_{1}, y_{1}, z_{1}\right),
\end{gathered}
$$

where

$$
\begin{gathered}
F_{1}\left(x_{1}, y_{1}, z_{1}\right)=k\left(-b^{2} x_{1}^{2}+\frac{\sqrt{b^{2}+a b}}{2} x_{1} y_{1}-\frac{a+b}{b} y_{1}^{2}\right) \\
F_{2}\left(x_{1}, y_{1}, z_{1}\right)=\frac{b^{2}}{\sqrt{b^{2}+a b}} F_{1}\left(x_{1}, y_{1}, z_{1}\right) \\
F_{3}\left(x_{1}, y_{1}, z_{1}\right)=\frac{b^{3}+b}{a} F_{1}\left(x_{1}, y_{1}, z_{1}\right)
\end{gathered}
$$

in which

$$
k=\frac{1}{a\left(b^{3}+b+a\right)} .
$$


In the following, we will follow the procedures proposed by Hassard et al. [18] to figure out the necessary quantities. One can get

$$
\begin{gathered}
g_{11}=\frac{1}{4}\left[\frac{\partial^{2} F_{1}}{\partial x_{1}^{2}}+\frac{\partial^{2} F_{1}}{\partial y_{1}^{2}}+i\left(\frac{\partial^{2} F_{2}}{\partial x_{1}^{2}}+\frac{\partial^{2} F_{2}}{\partial y_{1}^{2}}\right)\right] \\
=\frac{k\left(a+b+b^{3}\right)}{2 b}+i \frac{k\left(a b+b^{2}-b^{4}\right)}{2 \sqrt{b^{2}+a b}}, \\
g_{02}=\frac{1}{4}\left[\frac{\partial^{2} F_{1}}{\partial x_{1}^{2}}-\frac{\partial^{2} F_{1}}{\partial y_{1}^{2}}-2 \frac{\partial^{2} F_{2}}{\partial x_{1} \partial y_{1}}+i\left(\frac{\partial^{2} F_{1}}{\partial x_{1}^{2}}-\frac{\partial^{2} F_{1}}{\partial y_{1}^{2}}+2 \frac{\partial^{2} F_{2}}{\partial x_{1} \partial y_{1}}\right)\right] \\
=-\frac{k\left(b^{3}+2 a+2 b\right)}{4 b}-i \frac{k\left(2 b^{4}+3 b^{2}+3 a b\right)}{4 \sqrt{b^{2}+a b}}, \\
g_{20}=\frac{1}{4}\left[\frac{\partial^{2} F_{1}}{\partial x_{1}^{2}}-\frac{\partial^{2} F_{1}}{\partial y_{1}^{2}}+2 \frac{\partial^{2} F_{2}}{\partial x_{1} \partial y_{1}}+i\left(\frac{\partial^{2} F_{1}}{\partial x_{1}^{2}}-\frac{\partial^{2} F_{1}}{\partial y_{1}^{2}}-2 \frac{\partial^{2} F_{2}}{\partial x_{1} \partial y_{1}}\right)\right] \\
=-\frac{k\left(3 b^{3}+2 a+2 b\right)}{4 b}-i \frac{k\left(2 b^{4}+b^{2}+a b\right)}{4 \sqrt{b^{2}+a b}}, \\
G_{21}=\frac{1}{8}\left[\frac{\partial^{3} F_{1}}{\partial x_{1}^{3}}+\frac{\partial^{3} F_{1}}{\partial x_{1} \partial y_{1}^{2}}+\frac{\partial^{3} F_{2}}{\partial x_{1}^{2} \partial y_{1}}+\frac{\partial^{3} F_{2}}{\partial y_{1}^{3}}+i\left(\frac{\partial^{3} F_{2}}{\partial x_{1}^{3}}+\frac{\partial^{3} F_{2}}{\partial x_{1} \partial y_{1}^{2}}-\frac{\partial^{3} F_{1}}{\partial x_{1}^{2} \partial y_{1}}-\frac{\partial^{3} F_{1}}{\partial y_{1}^{3}}\right)\right]=0, \\
G_{110}=\frac{1}{2}\left[\frac{\partial^{2} F_{1}}{\partial x_{1} \partial z_{1}}+\frac{\partial^{2} F_{2}}{\partial y_{1} \partial z_{1}}+i\left(\frac{\partial^{2} F_{2}}{\partial x_{1} \partial z_{1}}-\frac{\partial^{2} F_{1}}{\partial y_{1} \partial z_{1}}\right)\right]=0, \\
G_{101}=\frac{1}{2}\left[\frac{\partial^{2} F_{1}}{\partial x_{1} \partial z_{1}}-\frac{\partial^{2} F_{2}}{\partial y_{1} \partial z_{1}}+i\left(\frac{\partial^{2} F_{2}}{\partial x_{1} \partial z_{1}}+\frac{\partial^{2} F_{1}}{\partial y_{1} \partial z_{1}}\right)\right]=0 .
\end{gathered}
$$

Then we have

$$
g_{21}=G_{21}+\left(2 G_{110} w_{11}+G_{101} w_{20}\right)=0
$$

where $w_{11}, w_{20}$ can be obtained by solving the following equations:

$$
\begin{gathered}
\lambda_{3} w_{11}=-h_{11}, \\
\left(\lambda_{3}-2 i \omega_{0}\right) w_{20}=-h_{20},
\end{gathered}
$$

in which

$$
h_{11}=\frac{1}{4}\left(\frac{\partial^{2} F_{3}}{\partial x_{1}^{2}}+\frac{\partial^{2} F_{3}}{\partial y_{1}^{2}}\right), \quad h_{20}=\frac{1}{4}\left(\frac{\partial^{2} F_{3}}{\partial x_{1}^{2}}-\frac{\partial^{2} F_{3}}{\partial y_{1}^{2}}-2 i \frac{\partial^{2} F_{3}}{\partial x_{1} \partial y_{1}}\right) \text {, }
$$

here, it is not necessary to calculate $w_{11}, w_{20}, h_{11}, h_{20}$ for obtaining the value of $g_{21}$. 
From the above analysis, one can compute the following quantities:

$$
\begin{aligned}
C_{1}(0)= & \frac{i}{2 \omega_{0}}\left[g_{20} g_{11}-2\left|g_{11}\right|^{2}-\frac{1}{3}\left|g_{02}\right|^{2}\right]+\frac{1}{2} g_{21} \\
= & \frac{11 b^{6}-35 b^{4}-35 a b^{3}+39 b^{2}-78 a b+39 a^{2}}{48 \omega_{0} a^{2} \sqrt{b^{2}+a b}} \\
& +i\left[\frac{16 b^{9}-37(b-a) b^{6}+(77 b+106 a-48) b^{4}}{96 \omega_{0} a^{2} b^{2}(a+b)\left(b^{3}+b+a\right)}\right. \\
& \left.\quad+\frac{\left(72 a^{2} b-36 b-120 a\right) b^{2}-\left(105 a^{2}+12\right) b-40 a^{3}}{96 \omega_{0} a^{2} b^{2}(a+b)\left(b^{3}+b+a\right)}\right], \\
\beta_{2}= & 2 \operatorname{Re} C_{1}(0) \\
= & \frac{11 b^{6}-35 b^{4}-35 a b^{3}+39 b^{2}-78 a b+39 a^{2}}{24 \omega_{0} a^{2} \sqrt{b^{2}+a b}}, \\
\mu_{2}= & \frac{-\operatorname{Re} C_{1}(0)}{\alpha^{\prime}(0)} \\
= & \frac{-(a+b)\left(b^{3}+a+b\right)\left(11 b^{6}-35 b^{4}-35 b^{3}+39 b^{2}-78 a b+39 a^{2}\right)}{24 a^{3} b^{4} \omega_{0}^{4}}, \\
\tau_{2}= & \frac{-\left(\operatorname{Im} C_{1}(0)+\mu_{2} \omega^{\prime}(0)\right)}{\omega_{0}} \\
= & \frac{2(a+b)\left(b^{3}+b+a\right)\left(11 b^{6}-35 b^{4}-35 a b^{3}+39 b^{2}-78 a b+39 a^{2}\right)}{96 \omega_{0}^{2} a^{2} b^{2}(a+b)} \\
& -\left[\frac{16 b^{9}-37(b-a) b^{6}+(77 b+106 a-48) b^{4}}{2} a^{2} b^{2}(a+b)\left(b^{3}+b+a\right)\right.
\end{aligned} .
$$

Now we can get the following theorem.

Theorem 4.2. System (2.2) exhibits a Hopf bifurcation at the equilibrium $O(0,0,0)$ as c passes through $c_{0}$, with the following properties:

(a) if $\mu_{2}>0(<0)$, the Hopf bifurcation is supercritical (subcritical) and the bifurcating periodic solutions exist for $c>c_{0}\left(<c_{0}\right)$;

(b) if $\beta_{2}<0(>0)$, the bifurcating periodic solutions are orbitally stable (unstable);

(c) if $\tau_{2}>0(<0)$, the period of bifurcating periodic solutions increases (decreases). 


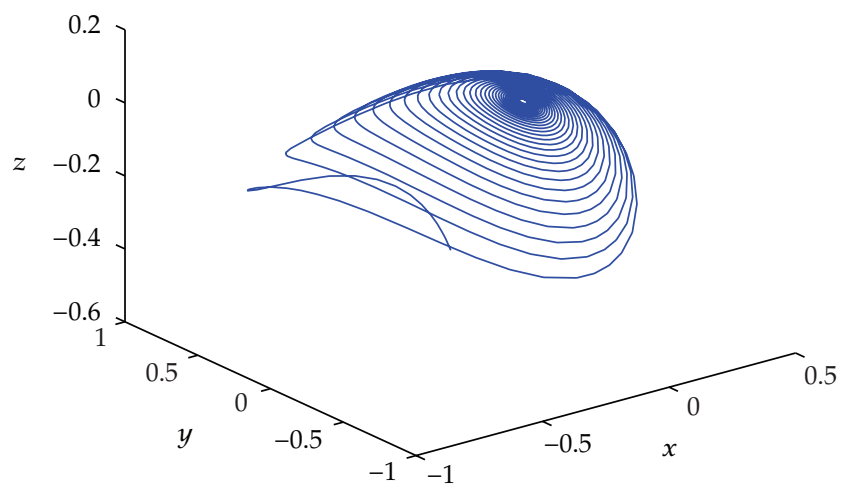

Figure 5: Phase diagram for system (2.2) with $c=-1.2$.

Remark 4.3. Here we only study the Hopf bifurcation about the equilibrium $O(0,0,0)$. For the Hopf bifurcation of system (2.2) at the equilibrium $E\left(c y_{0}, y_{0},-y_{0}\right)$, We first introduce the transformation $x=\bar{x}+c y_{0}, y=\bar{y}+y_{0}$, and $\mathrm{z}=\bar{z}-y_{0}$. Then, system (2.2) becomes

$$
\begin{gathered}
\dot{\bar{x}}=-\bar{y}-\bar{z}, \\
\dot{\bar{y}}=\bar{x}+c \bar{z}, \\
\dot{\bar{z}}=-(a+2 b) \bar{y}-b \bar{z}-\bar{y}^{2} ;
\end{gathered}
$$

hence, the discussion about the Hopf bifurcation of system (2.2) at the equilibrium $E\left(c y_{0}, y_{0},-y_{0}\right)$ is equivalent to the case of system $(4.16)$ at $(0,0,0)$. So the Hopf bifurcation of the equilibrium point $E\left(c y_{0}, y_{0},-y_{0}\right)$ can be treated similarly.

\subsection{Numerical Simulations}

When $a=0.5, b=1$, we can calculate $c=-1$ according to Theorem 4.1. It follows from the results in Section 4.2 that $\mu_{2}=21.5000, \beta_{2}=-3.5833, \tau_{2}=-0.1052$. In the light of Theorem 4.2, since $\mu_{2}>0$, the Hopf bifurcation is supercritical, which means that the equilibrium $O(0,0,0)$ of system (2.2) is stable when $c<c_{0}$ as shown in Figure 5. A Hopf bifurcation occurs when $c$ increases past $c_{0}$, that is, a family of periodic solutions bifurcate from the equilibrium, as shown in Figure 6. Since $\beta_{2}<0$, each individual periodic solution is stable. Since $\tau_{2}<0$, periods of bifurcating periodic solutions increase with increasing $c$. 


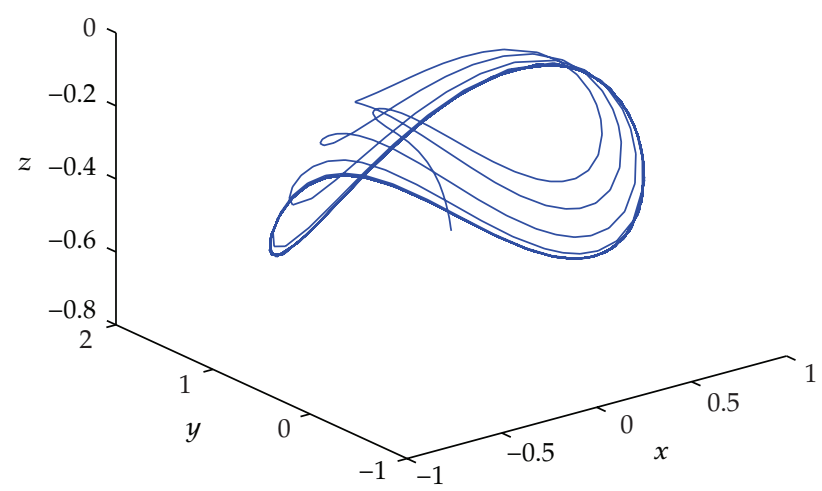

Figure 6: Phase diagram for system (2.2) with $c=-0.5$.

\section{Anticontrol of Hopf Limit Circles Based on MPS}

Next, we will design a stable Hopf limit circle into the Rössler system (2.1) via modified projective synchronization (MPS) proposed by [17]. To this end, the closed-loop control system based on MPS is formulated as follows:

$$
\begin{gathered}
\dot{x}=A x+f(x)+u, \\
\dot{y}=B y+g(y),
\end{gathered}
$$

where (5.1) denotes the response system and (5.2) stands for the drive system with a stable Hopf limit circle. $x=\left(x_{1}, x_{2}, \ldots, x_{n}\right)^{T}$ and $y=\left(y_{1}, y_{2}, \ldots, y_{n}\right)^{T}$ are state variables of the response system and the drive system, respectively. $A, B$ are matrices, whereas $f, g$ are nonlinear functions. The vector $u=\left(u_{1}, u_{2}, \ldots, u_{n}\right)^{T}$ represents the controller to be designed, with which the two systems can achieve synchronization by MPS. The symbols

$$
e_{i}=x_{i}-\alpha_{i} y_{i}, \quad i=1,2, \ldots, n
$$

stand for the errors between the state variables of the response system and those of the drive one. $\alpha=\operatorname{diag}\left(\alpha_{1}, \alpha_{2}, \ldots, \alpha_{n}\right)$ denotes the scaling factor matrix of the MPS, which can change the shape and size of the created Hopf limit circle. In order to achieve the modified projective synchronization, the controller $u$ for the active control [19] is chosen as

$$
u=-A \alpha y-f(x)+\alpha(B y+g(y))-P K e,
$$

where $P \in R^{n \times m}, K \in R^{m \times n}$, and $K$ is the feedback gain.

Then we obtain the error system

$$
\dot{e}=(A-P K) e,
$$


when the choice of $K$ makes the real parts of all eigenvalues of $(A-P K)$ negative by RouthHurwitz criterion, the errors $e_{i}(i=1,2, \ldots, n)$ exponentially converge to zero as time $t \rightarrow \infty$ and the MPS between the response system (5.1) and the drive system (5.2) occurs.

Remark 5.1. For a feasible control, the feedback gain $K$ can also be selected by the pole placement technique [20] such that the real parts of all eigenvalues of $(A-P K)$ are negative, when the controllability matrix is of full rank. In addition, $P$ can also be adjusted feasibly.

Remark 5.2. Notice that the values of the scaling factor components $\alpha_{i} \neq 0, i=1,2, \ldots, n$ have no effect on the controllability of the error system (5.5), because the eigenvalues of $(A-P K)$ are independent on the scaling factor matrix $\alpha$. This implies that we can arbitrarily adjust the scaling factor $\alpha_{i} \neq 0, i=1,2, \ldots, n$ to change the shape of the created Hopf limit circle without worrying about the robustness during control.

Now take the Rössler system (2.1) as the response system in which we want to create a stable Hopf limit circle. First, rewrite system (2.1) as follows:

$$
\begin{gathered}
\dot{x}_{1}=-x_{2}-x_{3}, \\
\dot{x}_{2}=x_{1} \\
\dot{x}_{3}=a\left(x_{2}-x_{2}^{2}\right)-b x_{3} .
\end{gathered}
$$

Note that the system is chaotic with parameters $(a, b)=(0.5,0.5)$ and the chaotic attractor is shown in Figure 1.

Next, choose the new Rössler-like system (2.2) with parameters $(a, b, c)=(0.5,1,-0.5)$ as the drive system, and rewrite it as follows:

$$
\begin{gathered}
\dot{y}_{1}=-y_{2}-y_{3}, \\
\dot{y}_{2}=y_{1}-0.5 y_{3}, \\
\dot{y}_{3}=0.5 y_{2}-y_{3}-y_{2}^{2} .
\end{gathered}
$$

According to dynamical analysis in Section 4, its stable Hopf limit circle surrounding the equilibrium $O(0,0,0)$ will appear, as shown in Figure 6. By the MPS scheme proposed above, the signal of the stable Hopf circle is employed to drive the Rössler system (5.6) to generate a stable Hopf circle surrounding the equilibrium $O(0,0,0)$.

In the controller $u$ as stated in (5.4), we have

$$
\begin{gathered}
A=\left(\begin{array}{ccc}
0 & -1 & -1 \\
1 & 0 & 0 \\
0 & 0.5 & -0.5
\end{array}\right), \quad f(x)=\left(\begin{array}{c}
0 \\
0 \\
-0.5 x^{2}
\end{array}\right), \\
B y+g(y)=\left(\begin{array}{c}
-y_{2}-y_{3} \\
y_{1}-0.5 y_{3} \\
0.5 y_{2}-y_{3}-y_{2}^{2}
\end{array}\right), \quad P=\left(\begin{array}{l}
0 \\
0 \\
1
\end{array}\right) .
\end{gathered}
$$


Let $K=\left(k_{1}, k_{2}, k_{3}\right)$, then

$$
A-P K=\left(\begin{array}{ccc}
0 & -1 & -1 \\
1 & 0 & 0 \\
-k_{1} & 0.5-k_{2} & -0.5-k_{3}
\end{array}\right)
$$

and the corresponding characteristic equation is

$$
\bar{f}(\lambda)=\lambda^{3}+\left(k_{3}+\frac{1}{2}\right) \lambda^{2}+\left(1-k_{1}\right) \lambda+k_{3}-k_{2}+1=0 .
$$

According to Routh-Hurwitz criterion, the real parts of the roots of (5.10) are all negative if and only if

$$
k_{3}+\frac{1}{2}>0, \quad k_{3}-k_{2}+1>0, \quad k_{2}-k_{1}\left(\frac{1}{2}-k_{3}\right)-\frac{1}{2}>0 .
$$

As long as the feedback gain $K=\left(k_{1}, k_{2}, k_{3}\right)$ satisfying condition (5.11) is chosen, the MPS between the response system (5.6) and the drive system (5.7) will be achieved according to the analysis above, that is, we can successfully generate a stable Hopf limit circle surrounding $O(0,0,0)$ in Rössler system (5.6) and the scaling factor $\alpha_{i} \neq 0, i=1,2,3$, can be arbitrarily adjusted to change the shape of the created Hopf circle.

Case 1. Choose $\alpha=\operatorname{diag}(1,2,3)$ and $K=(0,1,1)$. It is easy to verify that the feedback gain $K=\left(k_{1}, k_{2}, k_{3}\right)$ satisfies condition (5.11). The time evolution of the errors is shown in Figure 7. A stable Hopf limit circle surrounding $O(0,0,0)$ is created as shown in Figure 8.

Case 2. Choose $\alpha=\operatorname{diag}(-1,-1,-1)$ and $K=(-1,2,3)$. It is easy to verify that the feedback gain $K=\left(k_{1}, k_{2}, k_{3}\right)$ satisfies condition (5.11). The time evolution of the errors is shown in Figure 9. Another stable Hopf limit circle surrounding $O(0,0,0)$ is created as shown in Figure 10.

The above numerical simulation results are presented to illustrate the effectiveness of the MPS-based anticontrol of Hopf circles. Furthermore, the feedback gain $K=\left(k_{1}, k_{2}, k_{3}\right)$ can change the speed of convergence of the error system, and the scaling factor $\alpha_{i} \neq 0, i=1,2,3$, can be adjusted to change the shape and size of the created Hopf limit circle.

\section{Conclusions}

In this paper, a new Rössler-like system has been proposed and its dynamical behaviors are analyzed. By choosing an appropriate bifurcation parameter, a Hopf bifurcation occurs at the equilibrium point in this system when the bifurcation parameter exceeds a critical value. The direction of the Hopf bifurcation and the stability of bifurcating periodic solutions are analyzed in detail. Further, a stable Hopf limit circle is generated surrounding the equilibrium point in the response system (the original Rössler system) via a modified projective synchronization. Numerical simulations illustrated the effectiveness of the anticontrol of Hopf limit circles. There are still some unknown dynamical behaviors such as heteroclinic 


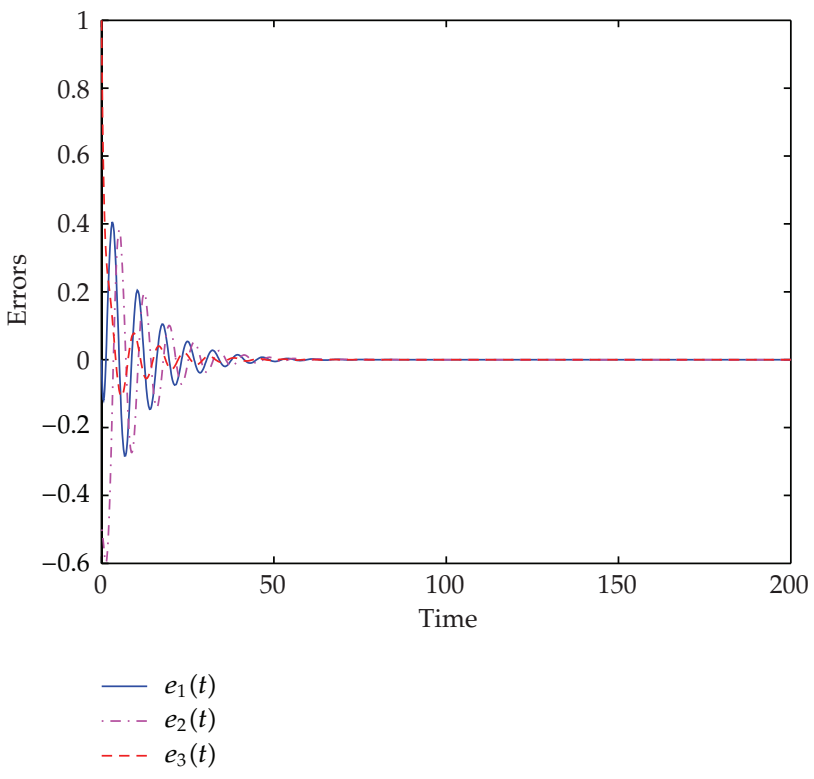

Figure 7: Synchronization errors in Case 1.

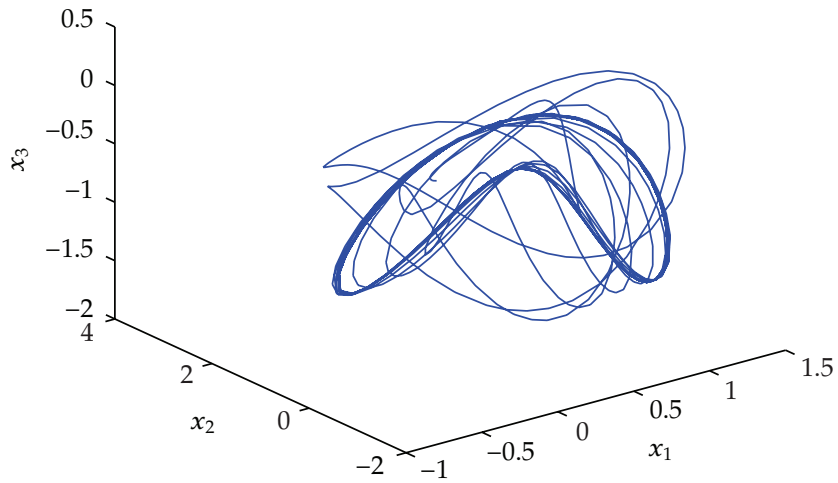

Figure 8: The created Hopf circle in Case 1.

(homoclinic) orbits about this system, as well as chaotic control to it, which deserve to be further investigated.

\section{Acknowledgments}

This work is supported by the National Natural Science Foundation of China (No. 610741 023), the Specialized Research Fund for the Doctoral Program of Higher Education of China (No. 20093401120001), the Natural Science Foundation of Anhui Province (No. 11040606M12) and the Natural Science Foundation of Anhui Education Bureau (No. KJ2010A035), and the 211 Project of Anhui University (No. KJJQ1102). 


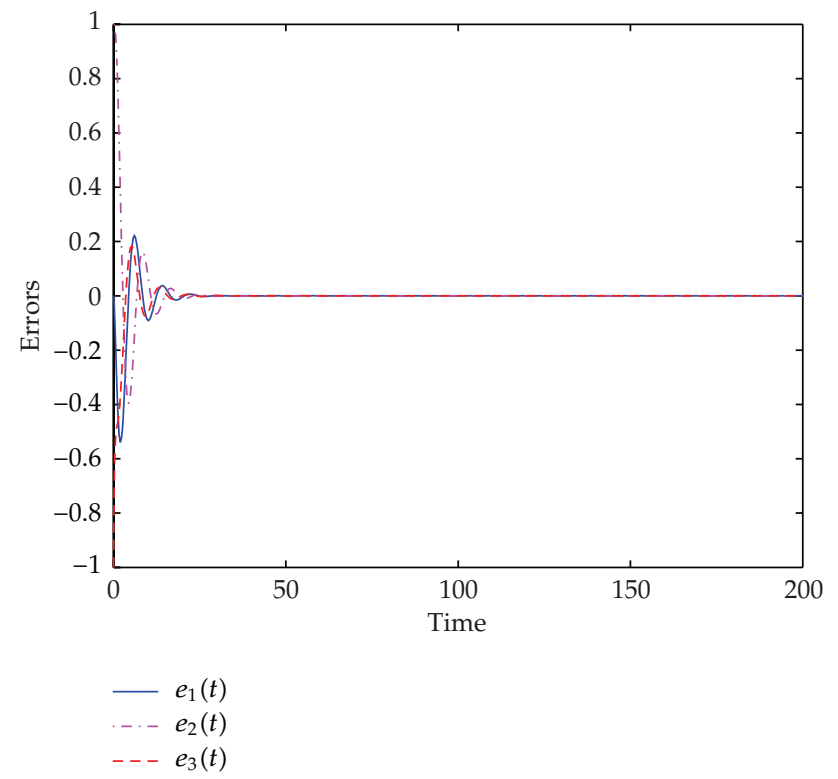

Figure 9: Synchronization errors in Case 2.

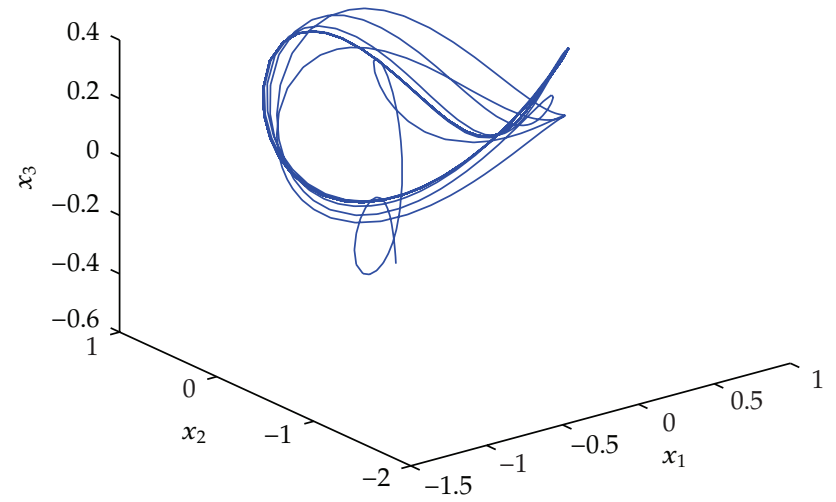

Figure 10: The created Hopf circle in Case 2.

\section{References}

[1] E. N. Lorenz, "Deterministic non-periodic flows," Journal of Atomic and Molecular Sciences, vol. 20, pp. 130-141, 1963.

[2] O. E. R. Rössler, "Continuous chaosfour prototype equations," Annals of the New York Academy of Sciences, vol. 316, pp. 376-392, 1979.

[3] X. Liao and P. Yu, "Study of globally exponential synchronization for the family of Rössler systems," International Journal of Bifurcation and Chaos, vol. 16, no. 8, pp. 2395-2406, 2006.

[4] Y. Liu and Q. Yang, "Dynamics of a new Lorenz-like chaotic system," Nonlinear Analysis, vol. 11, no. 4, pp. 2563-2572, 2010.

[5] G. Chen and T. Ueta, "Yet another chaotic attractor," International Journal of Bifurcation and Chaos, vol. 9, no. 7, pp. 1465-1466, 1999.

[6] J. Lü, G. Chen, and S. Zhang, "Dynamical analysis of a new chaotic attractor," International Journal of Bifurcation and Chaos, vol. 12, no. 5, pp. 1001-1015, 2002. 
[7] X. Zhou, Y. Wu, Y. Li, and Z. X. Wei, "Hopf bifurcation analysis of the Liu system," Chaos, Solitons and Fractals, vol. 36, no. 5, pp. 1385-1391, 2008.

[8] R. A. Van Gorder and S. R. Choudhury, "Analytical Hopf bifurcation and stability analysis of T system," Communications in Theoretical Physics, vol. 55, no. 4, pp. 609-616, 2011.

[9] Y. A. Kuznetsov, Elements of Applied Bifurcation Theory, vol. 112, Springer, New York, NY, USA, 1998.

[10] F. Dercole and S. Maggi, "Detection and continuation of a border collision bifurcation in a forest fire model," Applied Mathematics and Computation, vol. 168, no. 1, pp. 623-635, 2005.

[11] Y. Ma, M. Agarwal, and S. Banerjee, "Border collision bifurcations in a soft impact system," Physics Letters A, vol. 354, no. 4, pp. 281-287, 2006.

[12] J. Guckenheimer and P. Holmes, Nonlinear Oscillations, Dynamical Systems, and Bifurcations of Vector Fields, Springer, New York, NY, USA, 1983.

[13] C. C. Yu, Autotuning of PID Controllers: A Relay Feedback Approach, Springer, Berlin, Germany, 1999.

[14] Q. G. Wang, T. H. Lee, and C. Lin, Relay Feedback: Analysis, Identification and Control, Springer, London, UK, 2003.

[15] E. H. Abed and J.-H. Fu, "Local feedback stabilization and bifurcation control. I. Hopf bifurcation," Systems \& Control Letters, vol. 7, no. 1, pp. 11-17, 1986.

[16] P. K. Yuen and H. H. Bau, "Rendering a subcritical Hopf bifurcation supercritical," Journal of Fluid Mechanics, vol. 317, pp. 91-109, 1996.

[17] G.-H. Li, "Modified projective synchronization of chaotic system," Chaos, Solitons and Fractals, vol. 32, no. 5, pp. 1786-1790, 2007.

[18] B. D. Hassard, N. D. Kazarinoff, and Y. H. Wan, Theory and Applications of Hopf Bifurcation, Cambridge University Press, Cambridge, Mass, USA, 1982.

[19] G. Wen, "Designing Hopf limit circle to dynamical systems via modified projective synchronization," Nonlinear Dynamics, vol. 63, no. 3, pp. 387-393, 2011.

[20] P. N. Paraskevopoulos, Morden Control Engingneering, Marcel Dekker, New York, NY, USA, 2002. 


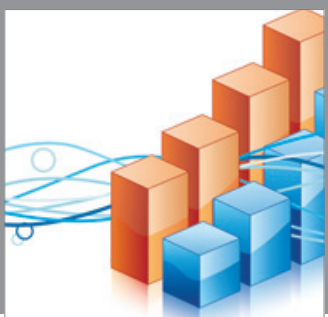

Advances in

Operations Research

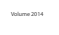

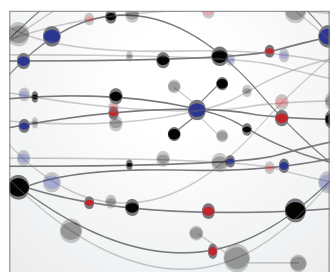

\section{The Scientific} World Journal
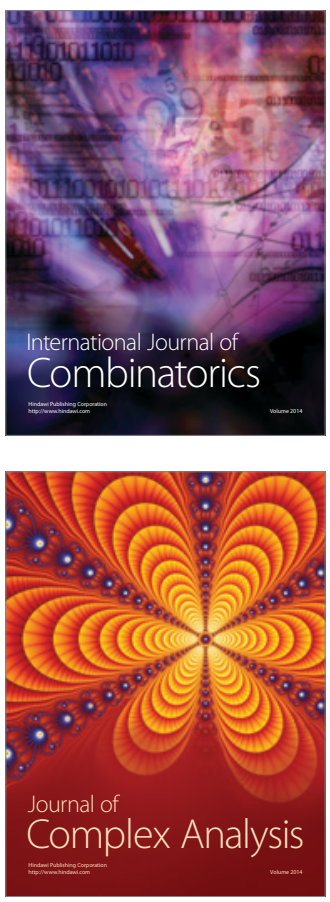

International Journal of

Mathematics and

Mathematical

Sciences
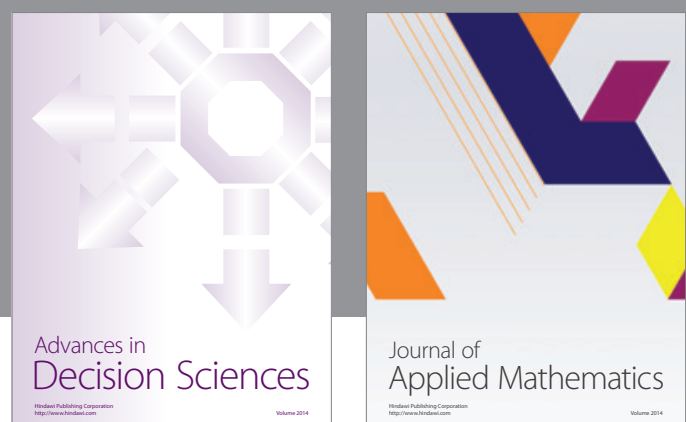

Journal of

Applied Mathematics
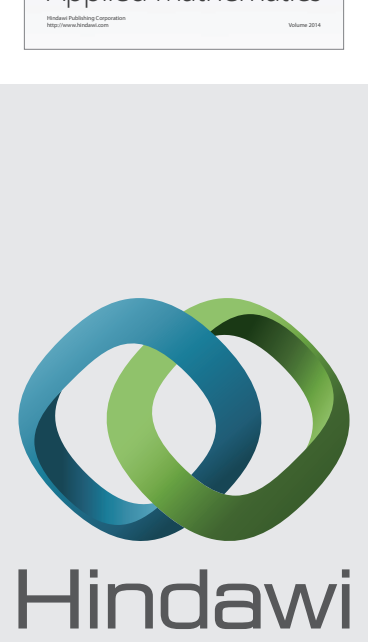

Submit your manuscripts at http://www.hindawi.com
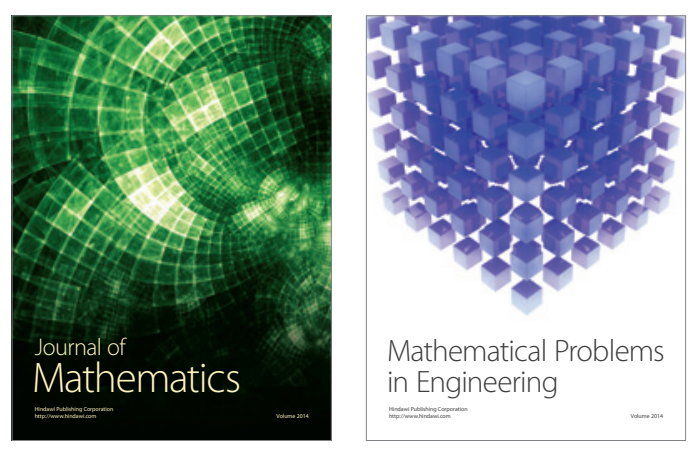

Mathematical Problems in Engineering
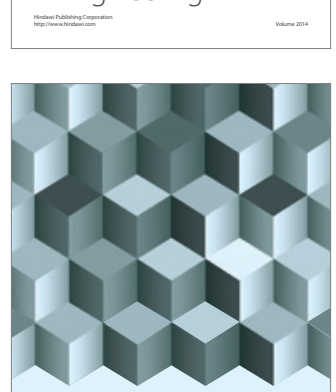

Journal of

Function Spaces
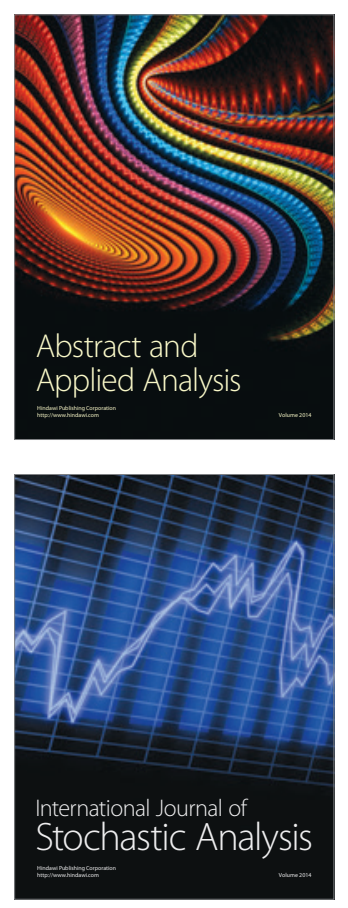

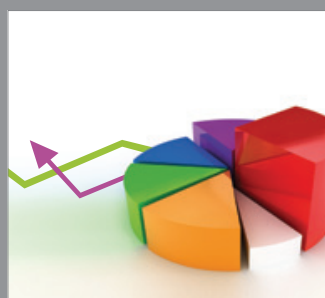

ournal of

Probability and Statistics

Promensencen
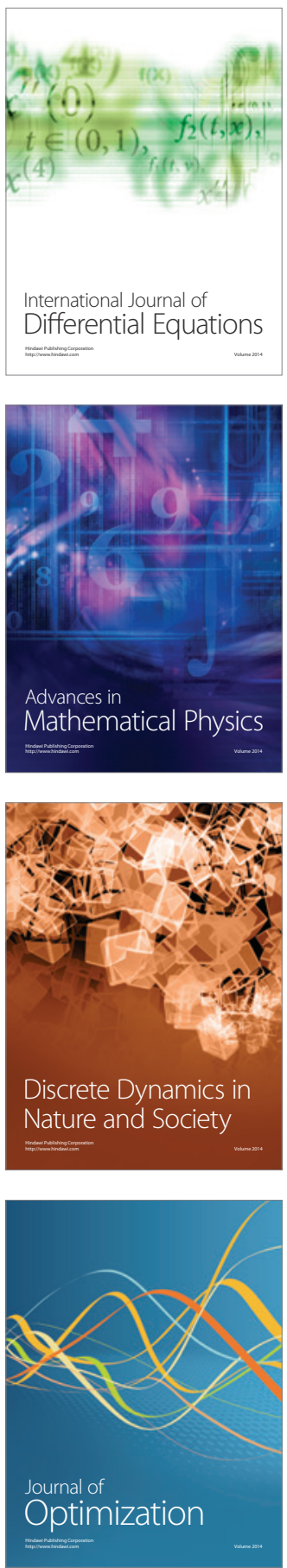